\title{
Dielectric breakdown at sub-critical fields
}

Cite as: Appl. Phys. Lett. 113, 112901 (2018); https://doi.org/10.1063/1.5044718

Submitted: 15 June 2018 . Accepted: 27 August 2018 . Published Online: 11 September 2018

Zhou Zuo (D, L. A. Dissado (D, N. M. Chalashkanov (D, S. J. Dodd, and Chenguo Yao

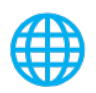

View Online

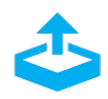

Export Citation

\section{ARTICLES YOU MAY BE INTERESTED IN}

Direct observation of the domain kinetics during polarization reversal of tetragonal PMN-PT crystal

Applied Physics Letters 113, 112902 (2018); https://doi.org/10.1063/1.5046657

Towards electrocaloric heat pump-A relaxor ferroelectric polymer exhibiting large electrocaloric response at low electric field

Applied Physics Letters 113, 113902 (2018); https://doi.org/10.1063/1.5048599

Solid-immersion metalenses for infrared focal plane arrays

Applied Physics Letters 113, 111104 (2018); https://doi.org/10.1063/1.5040395 


\title{
Dielectric breakdown at sub-critical fields
}

\author{
Zhou Zuo, ${ }^{1, a)}$ L. A. Dissado, ${ }^{2, a)}$ N. M. Chalashkanov, ${ }^{2}$ S. J. Dodd, ${ }^{2}$ and Chenguo Yao ${ }^{1, b)}$ \\ ${ }^{1}$ Department of High Voltage and Insulation Engineering, School of Electrical Engineering, \\ Chongqing University, Shapingba Main Street, No. 174, Chongqing 400044, China \\ ${ }^{2}$ Department of Engineering, University of Leicester, University Road, Leicester LE1 7RH, United Kingdom
}

(Received 15 June 2018; accepted 27 August 2018; published online 11 September 2018)

\begin{abstract}
We present a quantitative physical model based on experimentally determined parameter values for the description of the way that dielectric breakdown occurs after a period of stressing at fields below the critical values required for deterministic theories. It is found that for most of the material lifetime isolated regions of deterioration occur and that breakdown only ensues when the field in one such region reaches a critical value that produces a runaway extension. Only at low fields, do the deteriorated regions link up to form a conducting short-circuit. The generic form used means that the theory can be adapted for different physical mechanisms, and its concepts can be applied to the electrical reliability of many different types of dielectrics in a wide range of applications. Published by AIP Publishing. https://doi.org/10.1063/1.5044718
\end{abstract}

The insulating nature of a dielectric fails when a fielddriven process ${ }^{1-5}$ becomes unstable above a critical field. A field dependent equilibrium should occur at sub-critical fields, but instead the dielectric breaks down after a period of time. ${ }^{1,6}$ The nature of the changes involved, their generation mechanism, and the criteria for failure are a matter of debate. ${ }^{7-10}$ A number of approaches to dielectric breakdown due to ageing at sub-critical applied fields have been based on the way that conducting contaminants cause failure. These enhance the local field to a critical value and either link up to form a conducting path across the material ${ }^{1-13}$ or else generate damage that advances across the material in a tree-like structure as the high field is regenerated step by step. ${ }^{14,15}$ Thus, it has been suggested that ageing breakdown in the absence of such field-enhancing entities is the result of a field driven diffusion limited aggregation (DLA) of defects ${ }^{16,17}$ to form a conducting short circuit or alternatively a random field-driven extension of a growing conducting region, i.e., the dielectric breakdown model ${ }^{17-20}$ (DBM). A different approach has been to assume that chemical and physical changes driven by the electric field or high temperature degrade the dielectric until it can no longer function as an insulator ${ }^{7,10}$ or reach an extent at which an accelerating path to failure is initiated. ${ }^{8,9}$ In all the models proposed for ageing, there are various criteria for failure that are an inbuilt assumption of each model. Here, we use a quantitative ageing theory with verified generic parameter values ${ }^{21,22}$ for thin films to simulate ageing in thicker materials and hence determine the criterion for breakdown over a large range of sub-critical fields without any in-built assumptions as to its nature. Over most of the sub-critical field range, it is found that ageing breakdown is due to the formation of a few microscopic conducting regions ${ }^{22}$ enhancing the internal field to a critical value, a result that has not been suggested in previous ageing theories. However, at low ageing fields, the critical field cannot be reached before the regions link up

\footnotetext{
a) Authors to whom correspondence should be addressed: zhzuo@cqu.edu.cn and lad4@le.ac.uk

b)Electronic mail: yaochenguo@cqu.edu.cn
}

to form a conducting path across the material somewhat as suggested in the percolation theory. ${ }^{23}$

Our starting point is a modification of the chemical rate theory ${ }^{7}$ in which the reaction is made reversible as would be expected for physico-chemical changes in a solid where the "products" cannot be removed. Therefore, both driving and counterbalancing features are present as in the mechanisms that result in failure above a critical field. ${ }^{1-5}$ This rate equation previously used ${ }^{9,21,22}$ describes the changes produced by electrical ageing through the evolution of a normalized variable $\mathrm{X}(0 \leq \mathrm{X} \leq 1)$ defining the progress of the reaction

$$
\frac{\mathrm{dX}}{\mathrm{dt}}=\mathrm{k}_{\mathrm{f}}-\left(\mathrm{k}_{\mathrm{f}}+\mathrm{k}_{\mathrm{b}}\right) \mathrm{X} .
$$

The forward $\left(k_{f}\right)$ and backward $\left(k_{b}\right)$ rate constants are given in the following equations:

$$
\begin{gathered}
k_{f}=\frac{k T}{h} \exp \left(-\frac{H-C E^{b}}{k T}\right), \\
k_{b}=\frac{k T}{h} \exp \left(-\frac{H-\Delta}{k T}\right) .
\end{gathered}
$$

Here, $k$ is Boltzmann's constant, $h$ is Planck's constant, $H$ is the activation energy for the reaction, $\Delta$ is the energy difference between the unreacted and reacted states, and $C E^{b}$ is the energy available from the electric field $E$ to drive the changes. These equations predict an equilibrium state for the system at which $\mathrm{X}=A_{\text {eq }}=\left(k_{f}\left(k_{f}+k_{b}\right)\right)$, as would be expected for a mechanism in sub-critical fields. The application of an electric field changes the equilibrium state, and by assuming breakdown to occur when the reaction progress reached a critical extent $\mathrm{X}=A^{*}$, Eqs. (1)-(3) were used ${ }^{21}$ to yield lifetimes that agreed with the experimentally observed field and the temperature dependence of the lifetime of a number of insulating polymers. The characteristic values of the parameters $C$ and $A^{*}$ are independent of $E$ and $T$, but sample to sample variation in their value causes the observed lifetimes to be distributed. ${ }^{21,24}$ This fact allows us to construct a simulation of the electrical ageing of a large size 
sample by assuming that smaller elements of the same size $(36 \mu \mathrm{m})$ as those analyzed previously ${ }^{21}$ form the grid bonds of a matrix with bond values of $\mathrm{C}$ and $\mathrm{A}^{*}$ distributed according to their probability functions that fit the observed lifetime distribution of the small samples. In this way, the theory can be used to determine the progress of the internal changes that eventually lead to breakdown. Polyethylene terephthalate (PET) was chosen to illustrate the behavior because the electrical lifetime data analyzed previously ${ }^{21}$ were obtained using thin films that are therefore suitable for building a grid representing a section of a thick material. ${ }^{22}$ Each grid element is assigned the values of $\mathrm{C}$ and $\mathrm{A}^{*}$ at random ${ }^{22}$ such that the distribution of values in the simulation matrix obeys their fitted thin film distribution probabilities ${ }^{22,24}$ with the characteristic value of each distribution as previously determined. ${ }^{21}$ Since $\Delta \ll H$ in all $\operatorname{cases}^{21}(\Delta \sim 0.02 H$ for PET) and was shown to only influence the lifetimes in a small region of low fields, we have taken it to be zero here for the sake of simplicity.

In the simulations, sections of thick samples are represented by a $50 \times 402 \mathrm{D}$-grid with the grid bonds having the thickness $(36 \mu \mathrm{m})$ and the $\mathrm{C}$ and $\mathrm{A}^{*}$ parameter distributions as determined for the thin films and the thin film characteristic values $\left[\mathrm{C} / \mathrm{k}=1.582 \mathrm{~K}(\mathrm{MV} / \mathrm{m})^{-\mathrm{b}}\right.$ and $\left.\mathrm{A}^{*}=0.485\right]$. The other parameters, namely their field and temperature independent values as found from the thin films $(\mathrm{H} / \mathrm{k}=17762 \mathrm{~K}, \Delta=0$, and $\mathrm{b}=1.535$ ) since distributions in their value have not been found to occur, are given. ${ }^{24}$ Each bond represents a crosssection of a thin film with the vertical bonds relating to thin films oriented such that their thickness is parallel to the thickness of the simulated sample and the horizontal bonds corresponding to thin films whose thickness is perpendicular to that of the simulated sample. In this way, the simulated sample is composed of volume filling thin films, and the local fields determined during the simulation are always perpendicular to a thin film section across which they are constant. This is the situation for which the model parameters are known experimentally. The calculated field is resolved to the thickness of the thin film section to replicate the thin film situation for which experimental details are known. ${ }^{21}$ The model can therefore simulate the behavior of material samples thicker than the thin films used in its construction through the application of a potential difference to give a defined applied field.
A high DC voltage is applied to the top of the grid with the bottom at earth. Periodic boundary conditions are applied to the sides of the matrix, and ageing is confined to the inner $40 \times 40$ matrix to prevent any undue influence of the sides upon the outcome. The reaction progress, $X(t)$, is calculated for each bond according to its parameter values using Eq. (1) and the field in the bond. The changes, $\delta \mathrm{X}$, are accumulated over constant time steps $\delta \mathrm{t}$. The values chosen for $\delta \mathrm{t}$ depend upon the expected lifetime range and are different in different applied field ranges, with $\delta \mathrm{t}=1 \mathrm{~s}$ for $\mathrm{E} \leq 5 \mathrm{MV} / \mathrm{m}, \delta \mathrm{t}=10^{-3} \mathrm{~s}$ for $5<\mathrm{E} \leq 40 \mathrm{MV} / \mathrm{m}$, and $\delta \mathrm{t}=10^{-6} \mathrm{~s}$ for $\mathrm{E}>40 \mathrm{MV} / \mathrm{m}$. This is so that the number of time steps is adequate to describe the evolution of ageing damage with the values chosen using a rough estimate of the time required to fail the first bond in each of the given field ranges. When the reaction progress parameter $\mathrm{X}(\mathrm{t})$ in a bond reaches the critical extent, $\mathrm{A}^{*}$, assigned to it, the dielectric of the bond breaks down and becomes a conductor as would a thin film. ${ }^{21}$ The failed bond and any connected region thereof are taken to be an equipotential, and the field distribution is then updated using the finite difference method taken to self-consistency. The reaction progress in the un-failed bonds is then continued. The progress of the ageing deterioration is followed in this way until a conducting path is established across the matrix, and the simulated sample fails.

Figure 1 is typical of the simulations, which show that for about $90 \%$ of the lifetime, the local failures are isolated and a few failed regions then start to extend [Fig. 1(a)]. When an extended failure generates a critical value of the local field, Fig. 1(b), the extension starts an acceleration (runaway) to matrix failure [Fig. 1(c)]. This accords with the observation ${ }^{25}$ that very little detectable change occurs during ageing up to the instant of breakdown as previously noted. ${ }^{22}$ Unlike the fractal structures found ${ }^{18}$ in the DBM, the breakdown path is unbranched as expected for a deterministic runaway process ${ }^{15,26}$ except for regions near to the electrodes, where the extremely high fields and high field divergence at the extending tip allow the development of multiple branches particularly when these can link up existing failures near the main breakdown path. Plots of the maximum internal field (inset) and the number of failed bonds as a function of ageing time in Fig. 2 show that at the onset of runaway (accelerating) extension of a conducting region, the number of bond (a)

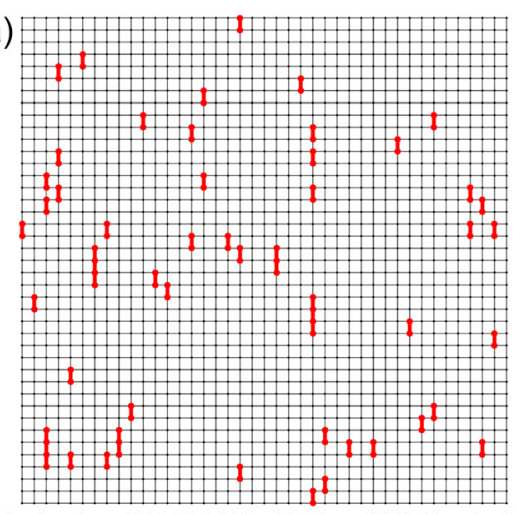

Ageing time: 1792473.3365 s Number of failed bonds: 57 (b)

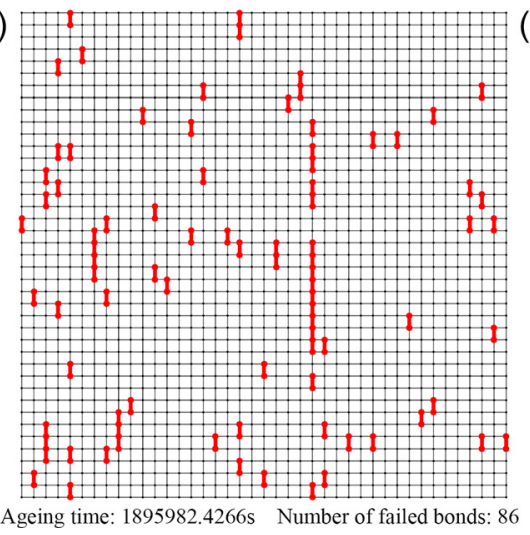

(c)

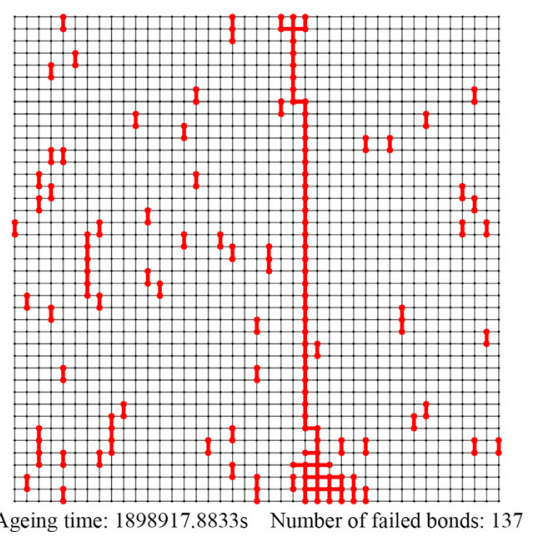

FIG. 1. The evolution of ageing at $\mathrm{E}=40 \mathrm{MV} / \mathrm{m}$ and $\mathrm{T}=383 \mathrm{~K}$, failed bonds shown in red: (a) when a few failed regions start to extend, (b) when extension starts to accelerate, and (c) at matrix failure. 


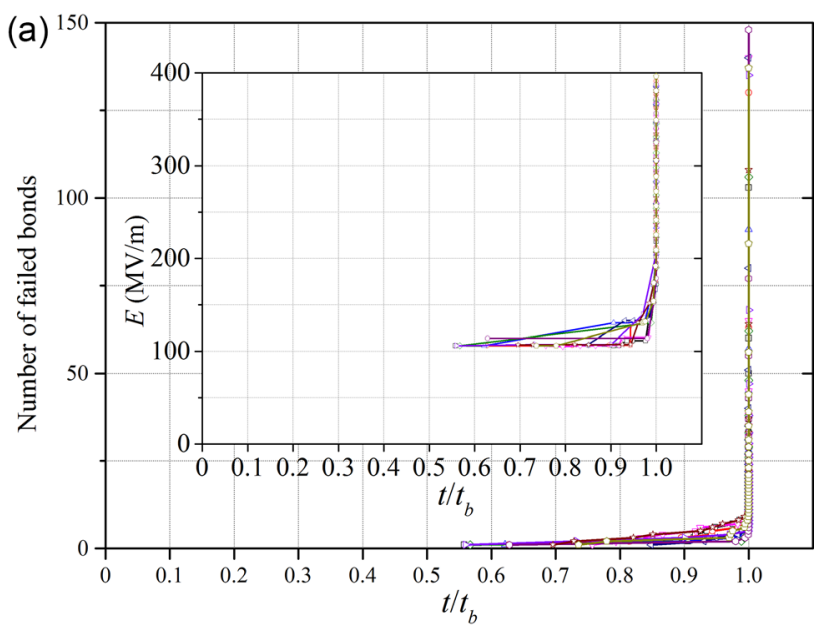

(b)

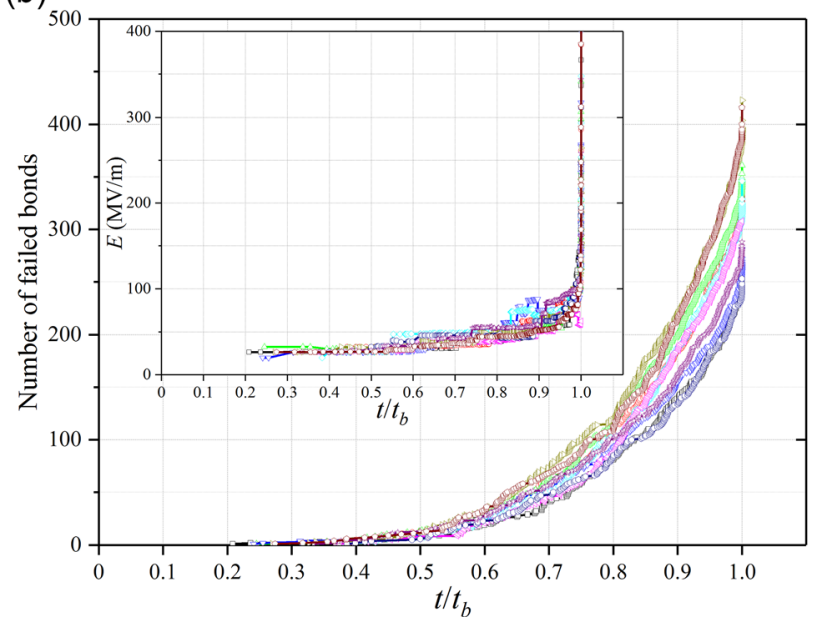

FIG. 2. Number of failed bonds and the maximum internal electric field (inset) as a function of time as a fraction of the breakdown time $t_{b}$ for repeated simulations with the same conditions: (a) $\mathrm{E}=80 \mathrm{MV} / \mathrm{m}, \mathrm{T}=383 \mathrm{~K}$ and (b) $\mathrm{E}=20 \mathrm{MV} / \mathrm{m}, \mathrm{T}=383 \mathrm{~K}$.

failures varies with the applied field. This is inconsistent with a percolation model ${ }^{23}$ for which a critical density of bond failures would be expected. However, the value of the maximum internal field when runaway occurs is near to the same irrespective of a fourfold difference in the applied field. The criterion for failure is therefore the generation of a critical value of the local field. The simulated lifetimes (Fig. 3) increase as the applied field reduces and fit the same theoretical lifetime expression, deduced from Eqs. (1)-(3), as the experimental data for thin films ${ }^{21}$ though with different characteristic values of the parameters. This change in parameter values, particularly the fitted value of $\mathrm{C}$, corresponds to a shift of the life line (line defining the relationship between the characteristic lifetime and the applied field) to shorter times at a given field for the thicker material. The value of the internal field required for the onset of runaway (termed runaway field in Fig. 3) is, however, independent of the applied field and can, therefore, be called a critical field since its generation determines the time to breakdown. The critical field is thus a material property as found from the analysis of the breakdown strength distribution of a system of conducting defects. ${ }^{13}$ Simulations on larger matrices or ones in which the width of the range of values assigned to $A^{*}$ and $C$

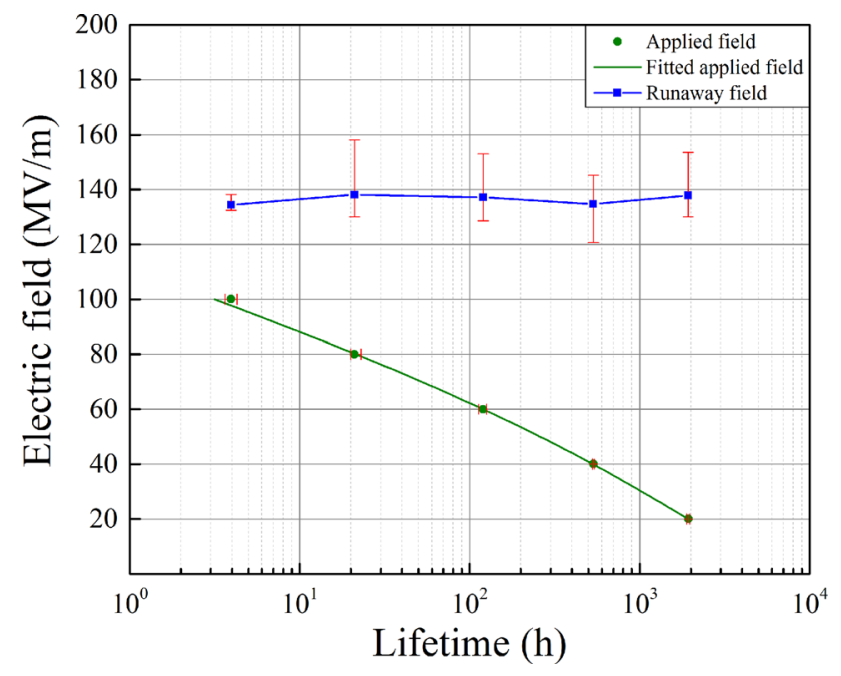

FIG. 3. Characteristic lifetimes as a function of the applied field and the internal maximum fields at the start of runaway (runaway field) for each applied field. The points are the characteristic values, $X_{c}$, in a Weibull distribution of variable $\mathrm{X}$ (lifetime or field) produced by ten repeated simulations: distribution density $=\mathrm{g}(\mathrm{X})=\beta \mathrm{X}^{\beta-1}\left(\mathrm{X}_{\mathrm{c}}\right)^{-\beta} \exp \left(-\left(\mathrm{X} / \mathrm{X}_{\mathrm{c}}\right)^{\beta}\right)$. The bars give the range of simulated values. The green line gives the fit to the life expression: ${ }^{21}$ Life $=L(E, T)=\left(\frac{h}{2 k T}\right) \exp \left(-\frac{H}{k T}\right) \exp \left(\frac{C E^{b}}{2 k T}\right)\left\{-\ln \left[\frac{A_{e q}-A^{*}}{A_{e q}}\right]\right\} / \cosh \left(C E^{b} / 2 k T\right)$ with $\mathrm{H} / \mathrm{k}=17762 \mathrm{~K}, \mathrm{~b}=1.535, \mathrm{~A}^{*}=0.432$, and $\mathrm{C} / \mathrm{k}=2.2 \mathrm{~K}(\mathrm{MV} / \mathrm{m})^{-\mathrm{b}}$.

has been changed or in which the variation in either $\mathrm{A}^{*}$ or $\mathrm{C}$ was eliminated confirm this contention by giving the same values. The critical field was, however, found to reduce with the increasing temperature.

In practice, most insulating materials experience fields in the range of $10-20 \mathrm{MV} / \mathrm{m}$ at which the generation of an internal critical field is the key criterion for breakdown. However, at fields of $5 \mathrm{MV} / \mathrm{m}$ or less, the behavior changes. It becomes impossible for the maximum internal field to reach the critical value. Instead, the number of failed bonds increases almost linearly with time [Fig. 4(a)] without any discernible increase in the production rate until they connect across the matrix, Fig. 4(b), in a manner similar to the percolation $^{23}$ and aggregation ${ }^{16}$ theories. Simulations using $80 \times 80$ and $160 \times 160$ matrices in the same fields show that this behavior applies even when the simulated material has a greater thickness and hence more chance to evolve the local field to the critical value. It is likely that in real insulation systems, the material would become unusable before this point is reached. ${ }^{7}$ In this event, however, a clear definition of an end-point is not generally available, and so, Fig. 4(b) represents a reasonable description of the state of the material as failure is approached at these fields.

Our approach is particularly appropriate to polymeric dielectrics since they are subject to local chemical and morphological changes; ${ }^{1,21}$ however, it could also be applied to crystalline and amorphous inorganic dielectrics which will possess a distribution of internal faults and imperfections that can grow by field driven changes in which an energy barrier is overcome. This would allow the concepts presented to be applied to dielectric layers in devices and power capacitors that experience applied fields of the magnitude shown in Fig. 3. Furthermore, Eq. (1) possesses a generic form that can, in principle, be related to the positive and negative feedback terms of the deterministic breakdown 


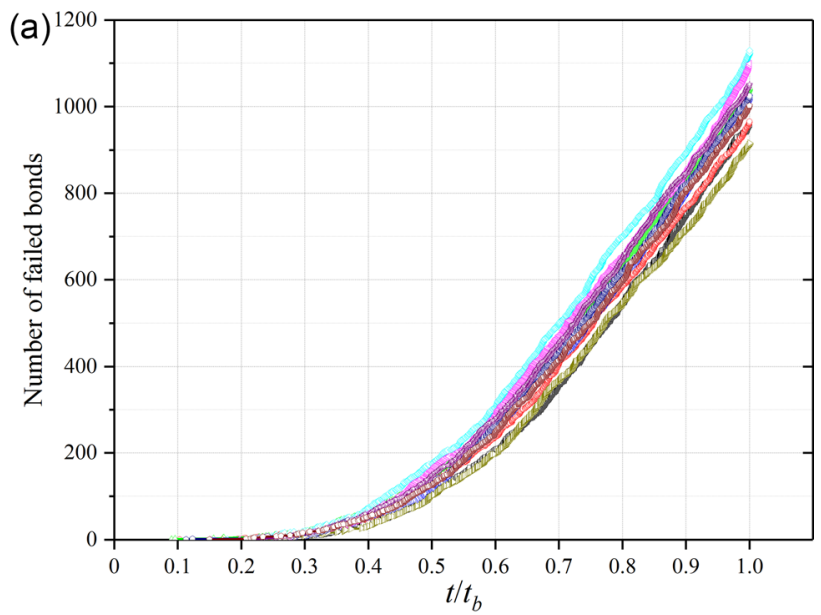

(b)

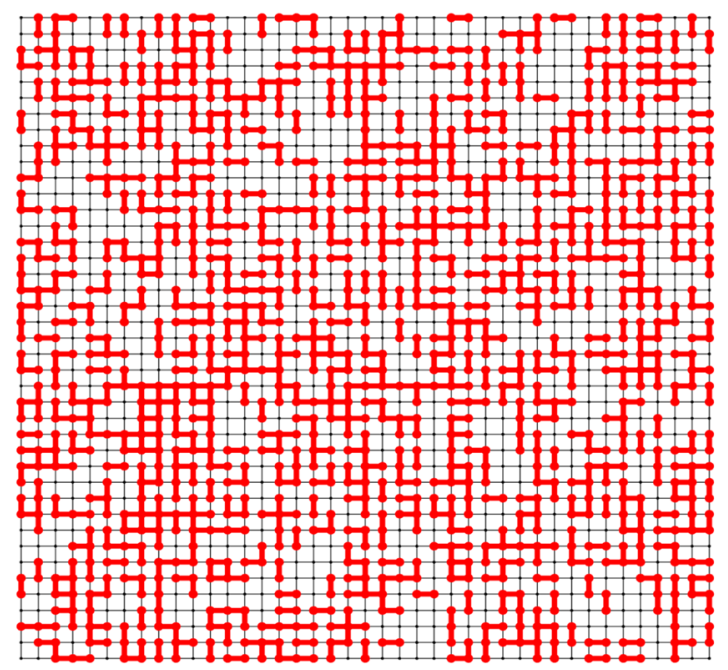

Ageing time: 16762083s Number of failed bonds: 1022

FIG. 4. Simulation of damage at $\mathrm{E}=5 \mathrm{MV} / \mathrm{m}$ and $\mathrm{T}=383 \mathrm{~K}$ : (a) number of failed bonds as a function of reduced time $\left(\mathrm{t} / \mathrm{t}_{\mathrm{b}}\right)$ and $(\mathrm{b})$ damage structure at breakdown.

theories ${ }^{1}$ with an appropriate choice of parameter values and their distribution. In this case, the material would come into equilibrium at sub-critical applied fields if the parameters had their characteristic value in every region. However, the spatial variation would allow the picture that we have presented to apply.

Our simulations allow each local region to behave as it would if it were an independent entity in a defined local field. In this way, electrical ageing is allowed to evolve according to the known behaviour of the local regions which become conducting after a certain time dependent upon the local values of a small number of material ageing parameters. The main result is that over most of the range of sub-critical fields, breakdown is an accelerating extension of damage whose onset is produced by the evolution of the local field to a critical value as a consequence of the formation of local conducting regions. Although such a breakdown criterion has been suggested for the breakdown strength of materials with a fixed density of pre-existing conducting inclusions, ${ }^{11-13}$ it has not previously been suggested as the criterion for ageing breakdown where the conducting regions are produced as a result of the ageing mechanism. A secondary point is that the evolution of the critical field is a group effect of all conducting regions and implies that models for ageing cannot be based on the development of degradation at a single isolated defect. Our other major result is that the criterion for failure changes at low fields to one in which the local regions link up to form a conducting short-circuit. This behaviour is closer to ageing breakdown as predicted by Dakin $^{7}$ or the percolation model ${ }^{23}$ than to the $\mathrm{DBM}^{17-20}$ and DLA $^{16}$ models. This change at low fields implies that the prediction of lifetimes at low service fields such as is commonly used may not be accurate.

Zhou Zuo and Chenguo Yao acknowledge the support of the National Natural Science Foundation of China Grant No. 51321063 for this work.

${ }^{1}$ L. A. Dissado and J. C. Fothergill, in Electrical Degradation and Breakdown in Polymers, edited by G. C. Stevens (IET, London, 1992).

${ }^{2}$ K. W. Wagner, AIEE J. 41, 1034 (1922).

${ }^{3}$ K. H. Stark and C. G. Garton, Nature 176, 1225 (1955).

${ }^{4}$ A. von Hippel, Ergeb. Exakten Naturwiss. 14, 79 (1935).

${ }^{5}$ H. Frohlich, Proc. R. Soc. London A160, 230 (1937).

${ }^{6}$ L. Simoni, IEEE Trans. Electr. Insul. 16, 278 (1981).

${ }^{7}$ T. W. Dakin, AIEE Trans. 67, 113 (1948).

${ }^{8}$ J. P. Jones, J. P. Llewellyn, and T. J. Lewis, IEEE Trans. Dielectr. Electr. Insul. 12, 951 (2005).

${ }^{9}$ G. Mazzanti, G. C. Montanari, and L. A. Dissado, IEEE Trans. Dielectr. Electr. Insul. 12, 876 (2005).

${ }^{10}$ C. Dang, J. L. Parpal, and J. P. Crine, IEEE Trans. Dielectr. Electr. Insul. 3, 237 (1996).

${ }^{11}$ P. M. Duxbury, P. D. Beale, and P. L. Leath, Phys. Rev. Lett. 57, 1052 (1986).

${ }^{12}$ P. D. Beale and P. M. Duxbury, Phys. Rev., B 37, 2785 (1988).

${ }^{13}$ R. W. Coppard, L. A. Dissado, S. M. Rowland, and R. Rakowski, J. Phys. Condens. Matter 1, 3041 (1989).

${ }^{14}$ L. A. Dissado, J. C. Fothergill, N. Wise, A. Willby, and J. Cooper, J. Phys. D: Appl. Phys. 33, L109 (2000).

${ }^{15}$ L. A. Dissado, IEEE Trans. Dielectr. Electr. Insul. 9, 483 (2002).

${ }^{16}$ T. A. Witten and L. M. Sander, Phys. Rev. Lett. 47, 1400 (1981).

${ }^{17}$ I. M. Irurzun, P. Bergero, V. Mola, M. C. Cordero, J. L. Vicente, and E. E. Mola, Chaos, Solitons Fractals 13, 1333 (2002).

${ }^{18}$ L. Niemeyer, L. Pietronero, and H. J. Wiesmann, Phys. Rev. Lett 52, 1033 (1984).

${ }^{19}$ H. J. Wiesmann and H. R. Zeller, J. Appl. Phys. 60, 1770 (1986).

${ }^{20}$ P. Bergero, F. Peruani, G. Solovey, I. M. Irurzun, J. L. Vicente, and E. E. Mola, Phys. Rev. E 69, 016123 (2004).

${ }^{21}$ L. A. Dissado, G. Mazzanti, and G. C. Montanari, IEEE Trans. Dielectr. Electr. Insul. 4, 496 (1997).

${ }^{22}$ L. A. Dissado and A. Thabet, J. Phys. D: Appl. Phys. 41, 085412 (2008).

${ }^{23}$ K. Wu, L. A. Dissado, and T. Okamoto, Appl. Phys. Lett. 85, 4454 (2004).

${ }^{24}$ L. A. Dissado, IEEE Trans. Dielectr. Electr. Insul. 9, 860 (2002).

${ }^{25}$ L. Markey and G. C. Stevens, J. Phys. D: Appl. Phys. 36, 2569 (2003).

${ }^{26}$ T. Mizutani, I. Kanno, M. Hikita, M. Ieda, and G. Sawa, IEEE Trans. Electr. Insul. 22, 473 (1987) 